\title{
Triboacoustic diagnostic fixed joints of machines
}

\author{
Aaleksandr Dykha ${ }^{1, *}$, and Sergij Matyukh ${ }^{1}$ \\ ${ }^{1}$ Khmelnitskyi National University, 29016, Instytutska 11, Ukraine
}

\begin{abstract}
The experimental results of the tribo-acoustic control in real time the integrity of nominally stationary friction joints under conditions of fretting are presented. The disturbance in the integrity of the tribosuppression as a result of wear has been reliably detected both by the change in the shape of the acoustic histograms of the operating noise and by the change in the noise spectrum due to the appearance of strong midfrequency modes of the contact gap. The appearance of two oppositely directed wave energy cascades in the regime of fretting has been observed: a direct cascade from mod the pump in the direction of high frequencies and the opposite - side to the low infrasonic frequencies. The former has been associated with microplastic deformations in the zones of actual contact, while the latter has been associated with the dynamic generation of slow angular motions in the friction system.
\end{abstract}

\section{Introduction}

Modern technological conditions of production activity present increased requirements to the accuracy and efficiency of measurements, to the quality of control and diagnostics of various aggregates at the stage of their design, manufacturing, testing, operation and repair. Traditional methods of non-destructive testing are widely used at the manufacturing and repair stage. However, when used in a working environment, there are significant difficulties. Acoustic methods, being one of the most informative, provide, on the one hand, the identification of various defects of the object of control, and, on the other hand, the ability to carry out operational measurements of the technological parameters of various processes. Modern development of analog and digital technology allows to significantly increase the sensitivity and accuracy of acoustic measurements, to expand the possibilities of their application for diagnostics and measurement of parameters of dynamic objects and processes.

In tribotechnics, acoustic methods have been widely used for analysis and to control friction [1-0]. The acoustoemission of the ultrasonic frequency range using special contacttype sensors has become the most widely employed $[1,3,4,8]$. Contactless acoustic control of sound and near infrasonic frequency bands based on standard or special software and hardware is much easier in technical implementation. However, it requires an additional analysis to isolate the useful signal against background noise. In the past, triboacoustics has primarily been used for mobile functional compounds [2, 5, 6]. It should seem that fretting in nominally motionless joints is hard to hear in the overall operating noise of an installation, mechanism, or machine. However, due to the mutual dependence of the constituents of dynamic processes proceeding in a complex technical system, a disturbance in the integrity of any such joints will inevitably affect the amplitude level and the spectral distribution of the operating noise. Due to the relatively low slip rates in fretting modes, the characteristic frequencies of acoustic emission fall precisely into the acoustic and near

\footnotetext{
* Corresponding author: tribosenator@gmail.com
} 
infrasonic range of the spectrum that enables using conventional sound recording equipment and standard computer software.

The aim of this work is to perform the contactless triboacoustic control of the integrity of a nominally motionless friction joint under fretting conditions, as well as to determine the nature and direction of the major wave processes of energy and momentum transfer in real time.

\section{Experimental}

The experimental setup used in our study for fretting tests has been described in detail elsewhere [10]. It does not contain rotating elements that would create an increased level of contact noise (Figs. 1, 2). According to the plane-ball scheme, fretting is studied with contact between unhardened steel 30 HGSA (the leading upper sample 1 in Fig. 1) with a ball of the LH-15 steel bearing with a diameter of $12 \mathrm{~mm}$ (Fig. 1, lower driven counterbody 14). The sample was preliminarily ground and subsequently polished to a surface roughness of the 9th class $(\mathrm{Ra}=0.2-0.3 \mu \mathrm{m})$. The reciprocating motion of the leading sample was produced using an electromagnet and a spring system. The amplitude of its vibrodisplacement Hs was $20 \mu \mathrm{m}$. The driven counterbody (ball) was clamped in the frame of the tensor bar (Fig. 2). Two-thirds of the normal contact load in the joint $(\sim 50 \mathrm{~N})$ was due to the weight of the drive and one-third was due to the torque wrench force (Fig. 1) that gradually attenuated during the fretting. The main working frequency of vibrations was 100 $\mathrm{Hz}$.

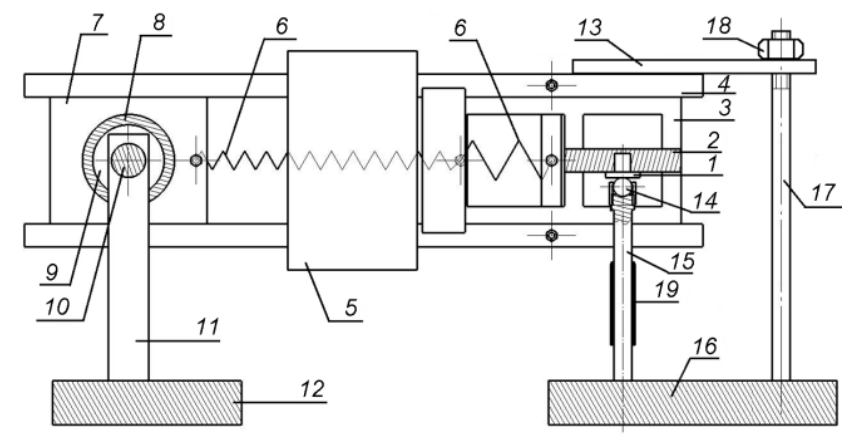

Fig. 1. Below the figure. Schematic of the experimental setup: (1) leading sample of a pair of friction; (2) sample holder; (3) carriage; (4) guides; (5) electromagnet; (6) a system of springs; (7) plates; (8) the bushing; (9) radial-thrust bearings; (10) shaft; (11) shaft supports; (12) base; (13) clamping plate; (14) driven counterbody (ball); (15) stretch bar; (16) base; (17) hairpin; (18) nut; (19) strain gages.

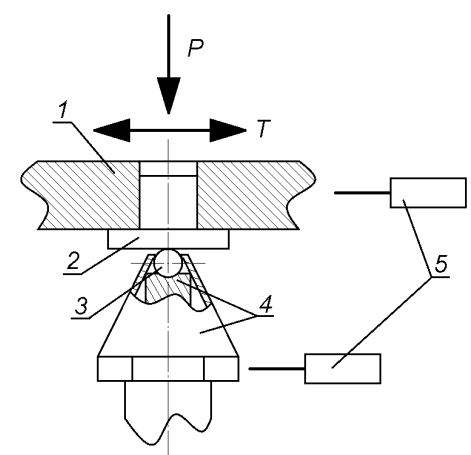

Fig. 2 Contact diagram: (1) sample holder; (2) sample; (3) counterbody (ball); (4) locking system counterbody; (5) inductive proximity sensors; $P$ is clamping force, $T$ is tangential force. 
To record the acoustic signal, a portable microphone Media-Tech SFX MT 383 microphone installed at a distance of several decimeters from the friction unit was used. Data were processed using the Audacity 2.0.4 program. Online plotting of the contact hysteresis loop in coordinates of the displacement of the driven counterbody versus the tangential force was used as an additional informative channel. The displacement and tangential force were measured using an inductive microdisplacement sensor and a bridge of strain gages on the beam, respectively. The acoustograms were prerecorded in the idle ode of the setup operation, i.e., with an almost vertical position of the drive guides. Then, in their horizontal position of the drive guides, the joint was initially lamped while tests were performed in the fretting mode, i.e., from the initial run-in process to the disturbance in the joint integrity resulted in a qualitative hanged in both the level of the amplitude and the spectral composition of the acoustic signal.

\section{Results and Discussion}

Figure 3 shows the acoustograms recorded for $1 \mathrm{~min}$ each as the testing develops. Acoustograms are useful for studying fretting in general.

(a)

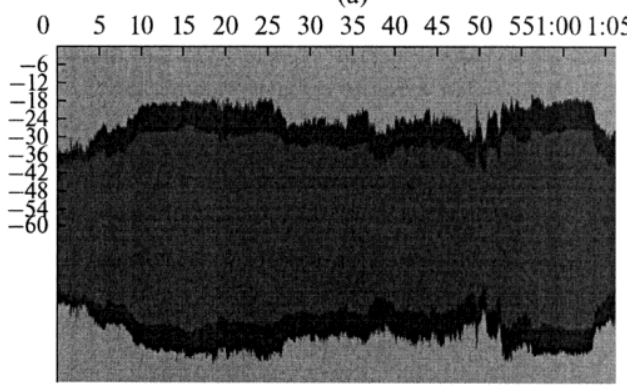

(c)

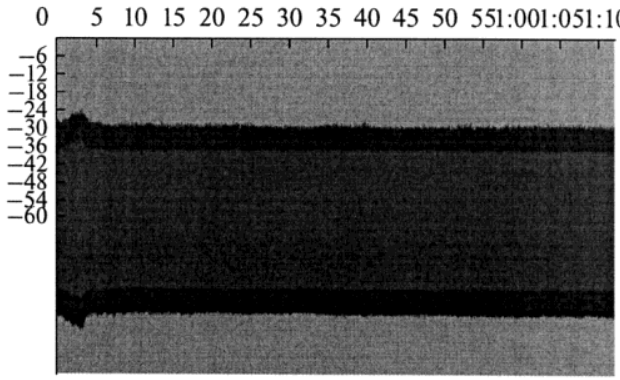

(b)

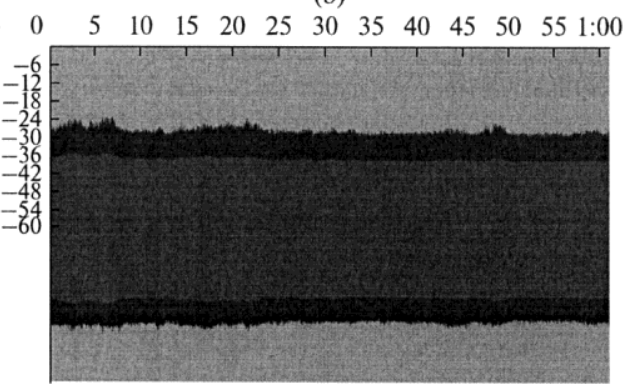

(d)

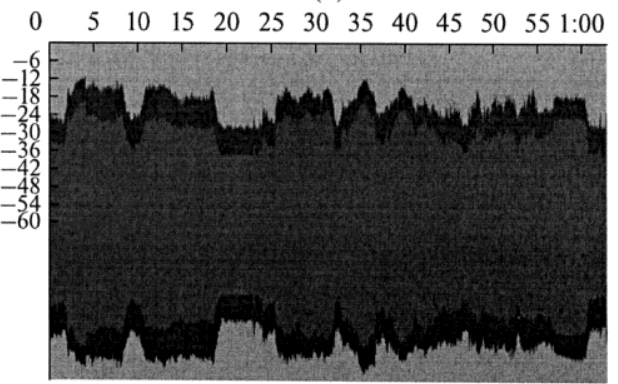

Fig. 3. Acoustograms of working noise recorded at (a) 1st, (b) 15 th, (c) 55 th, and (d) 88th min. Horizontal axis represents time in minutes and seconds and the vertical axis represents the signal level in $\mathrm{dB}$.

The low-frequency instability that was specific to the initial stage of run in (Fig. 3a) decreased significantly in about $1 \times 105$ cycles of oscillations (Fig. 3b), leading to a longer period of optimal joint operation $(\sim 5 \times 105$ cycles) (Fig. 3c). A notable disturbance in the integrity of the joint occurred after approximately $88 \mathrm{~min}$ of testing $(\sim 5.3 \times 105$ cycles $)$ (Fig. 3d).

The corresponding hysteresis loops were more similar, but less informative (Fig. 4). Nevertheless, at the final stage (Fig. 4d), the increase in amplitudes of high-frequency signal components was noticeable and followed by a decrease in the tangential force on the driven counterweight and a decrease in the amplitude of its oscillations due to slippage in the joint contact. 
(a)

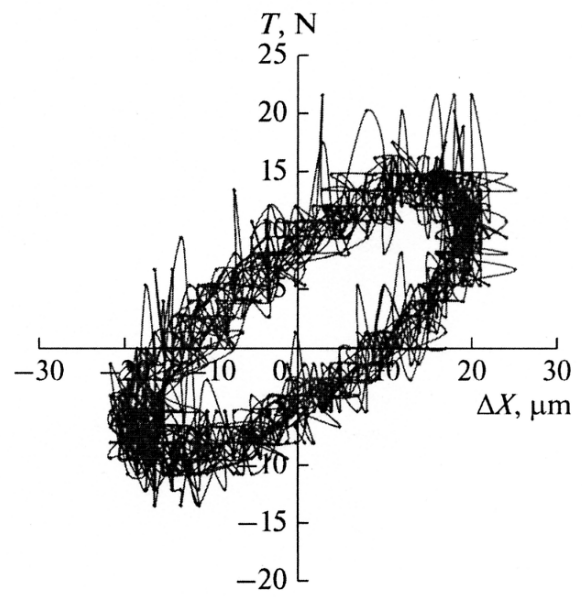

(c)

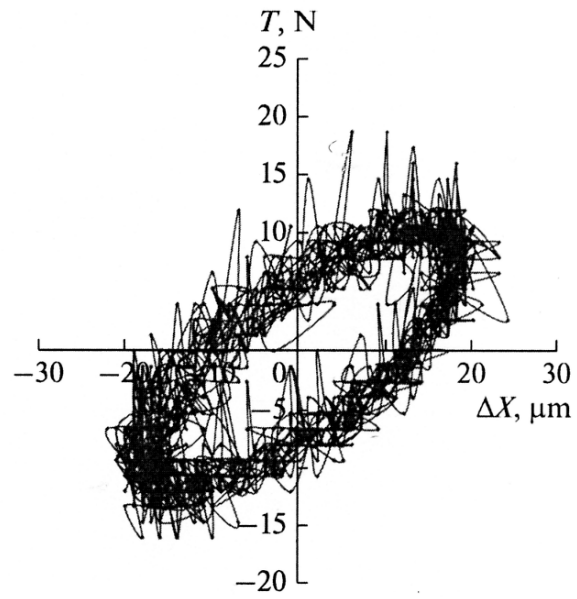

(b)

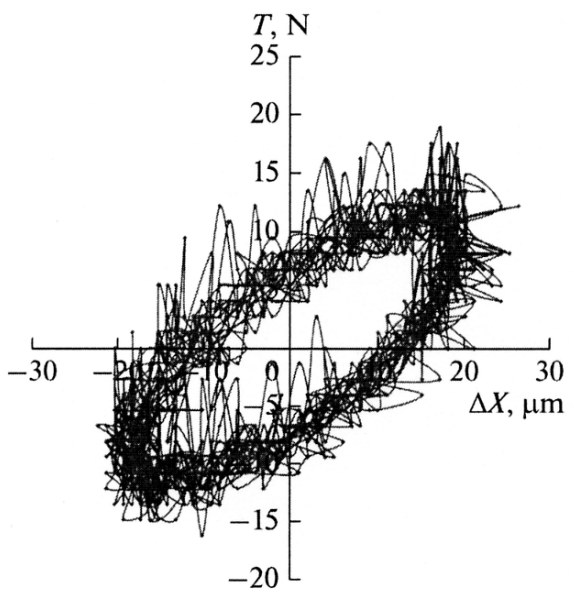

(d)

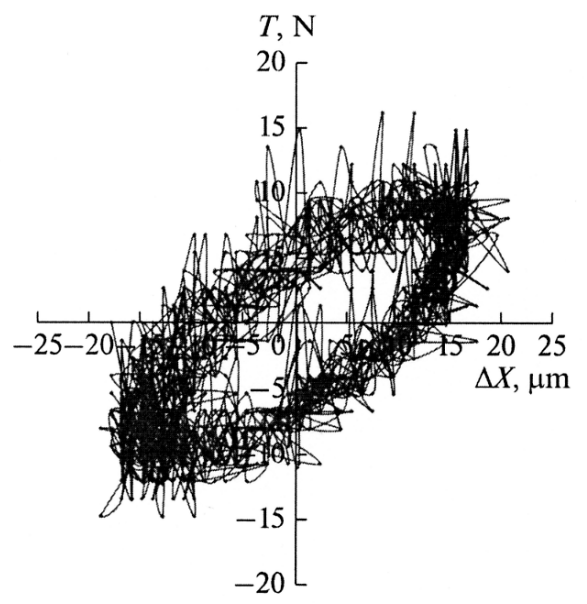

Fig. 4. Contact hysteresis loops plotted for (a) 1st, (b) 15th, (c) 55th, and (d) 88th min.

Of greatest interest in this case, the Fourier spectra of the above ecostorm - Fig. 5. They are dominated by two main pump frequency disturbances, $50 \mathrm{~Hz}$ and $100 \mathrm{~Hz}$ and their harmonics arising from the nonlinearity in a dynamic system and a corresponding disharmony of vibrations. These harmonics at the initial stage of running-in (Fig. 5a) form a well-defined direct energy cascade towards high frequencies with the maximum at frequencies of $1-5 \mathrm{kHz}$.

The occurrence of this maximum is due to quasi-normal contact vibrations induced by the processes of microplastic deformations at the stage of running-in. The other - the reverse energy cascade is directed from the pumping modes in the low frequency nearinfrasound region of the spectrum (Fig. 5a). Its origin is connected with guided slow angular movements of the guides of the actuator, which has a direct impact on the rapid vibration of the sample leading to friction and, consequently, on the processes of contact interaction in the zone of fretting.

This kind of low-frequency self-modulation of the friction process often is at the expense of cyclical damage accumulation and separation of wear particles [6]. In this case, such cyclicity is a consequence, not the cause of automodulation arising from the activation 
of the available degrees of freedom and feedback in the overall dynamic system of friction. Thus, the friction acts as a global (system-wide) and not just local (mesophysical) process.

In addition, we analyzed the Fourier spectra of the above-mentioned acoustograms and confirmed the qualitative change in the spectral composition of the acoustic emission of friction, which was associated with a disturbance in the joint integrity.

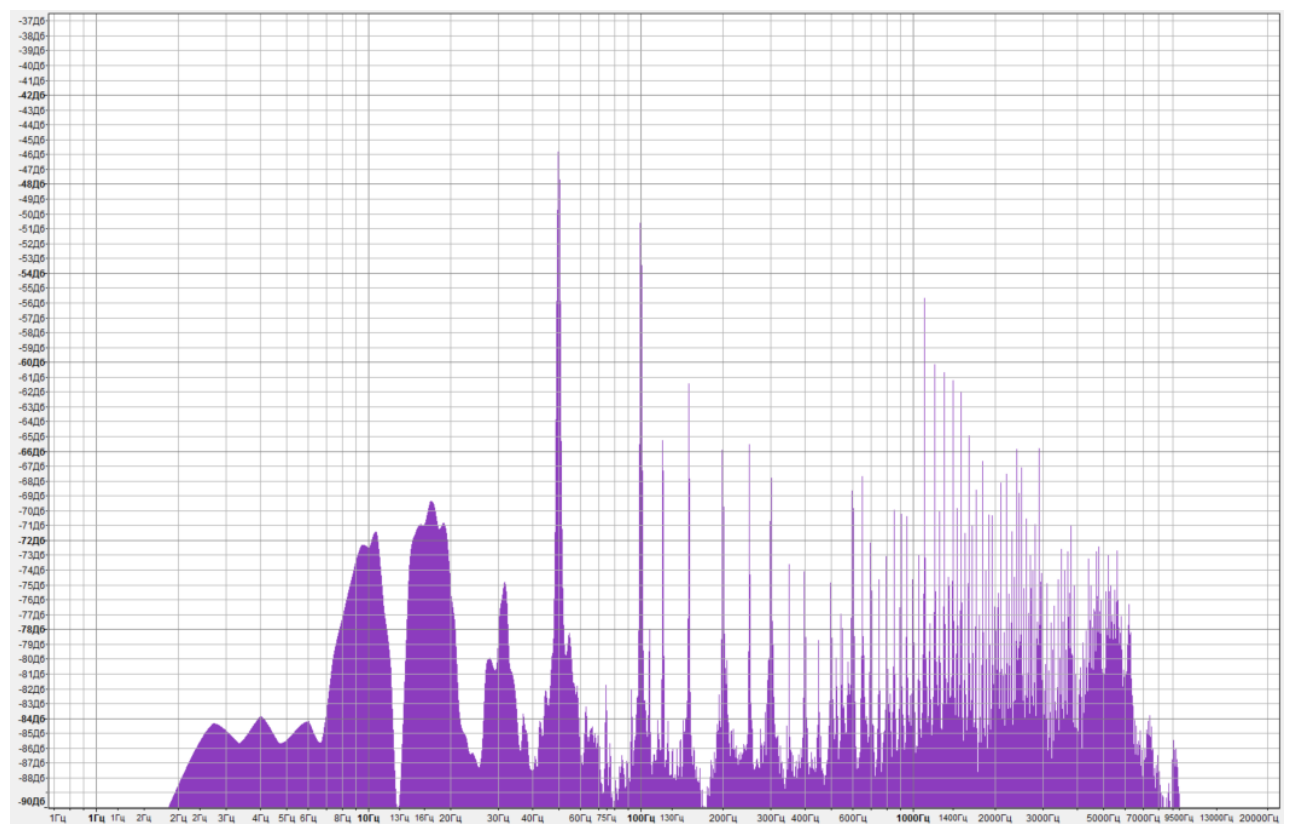

a

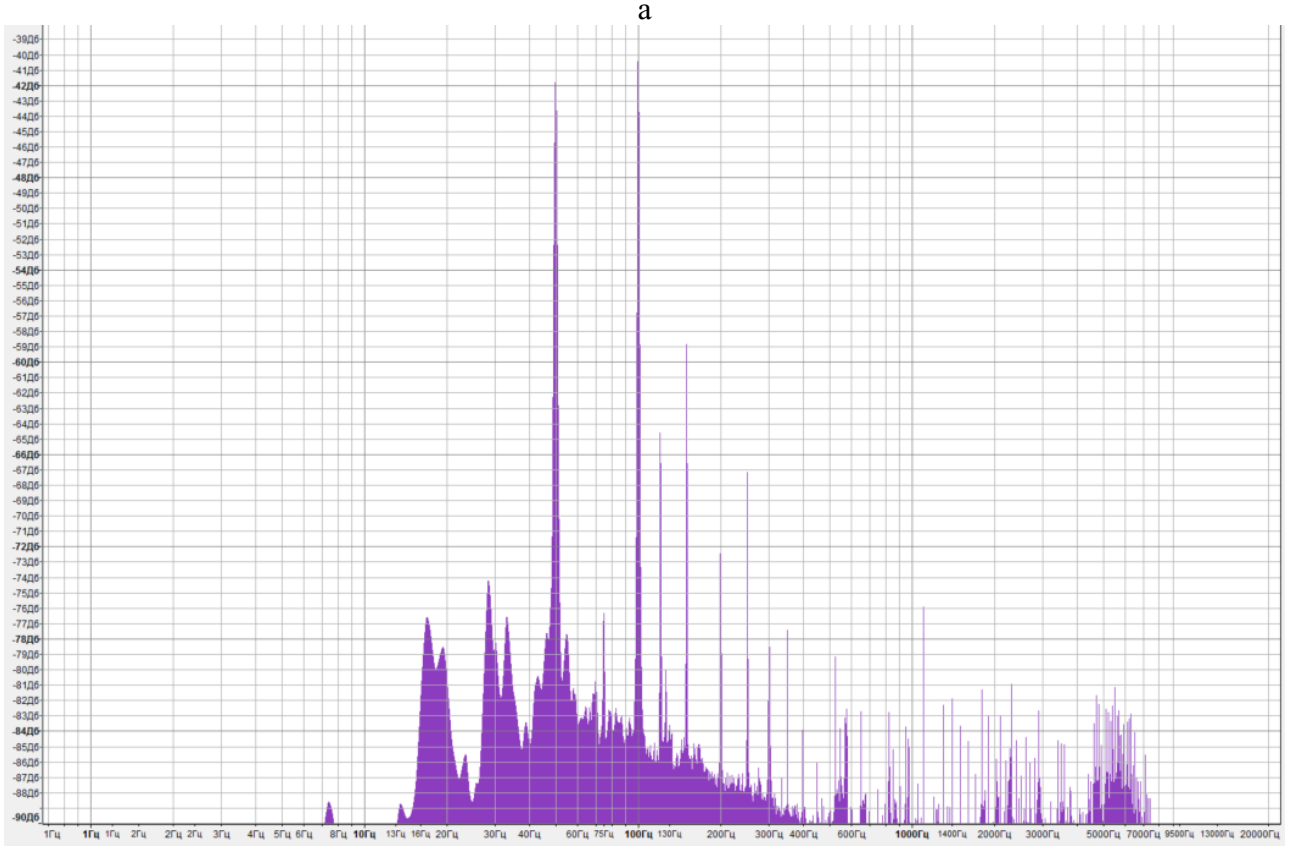

b 


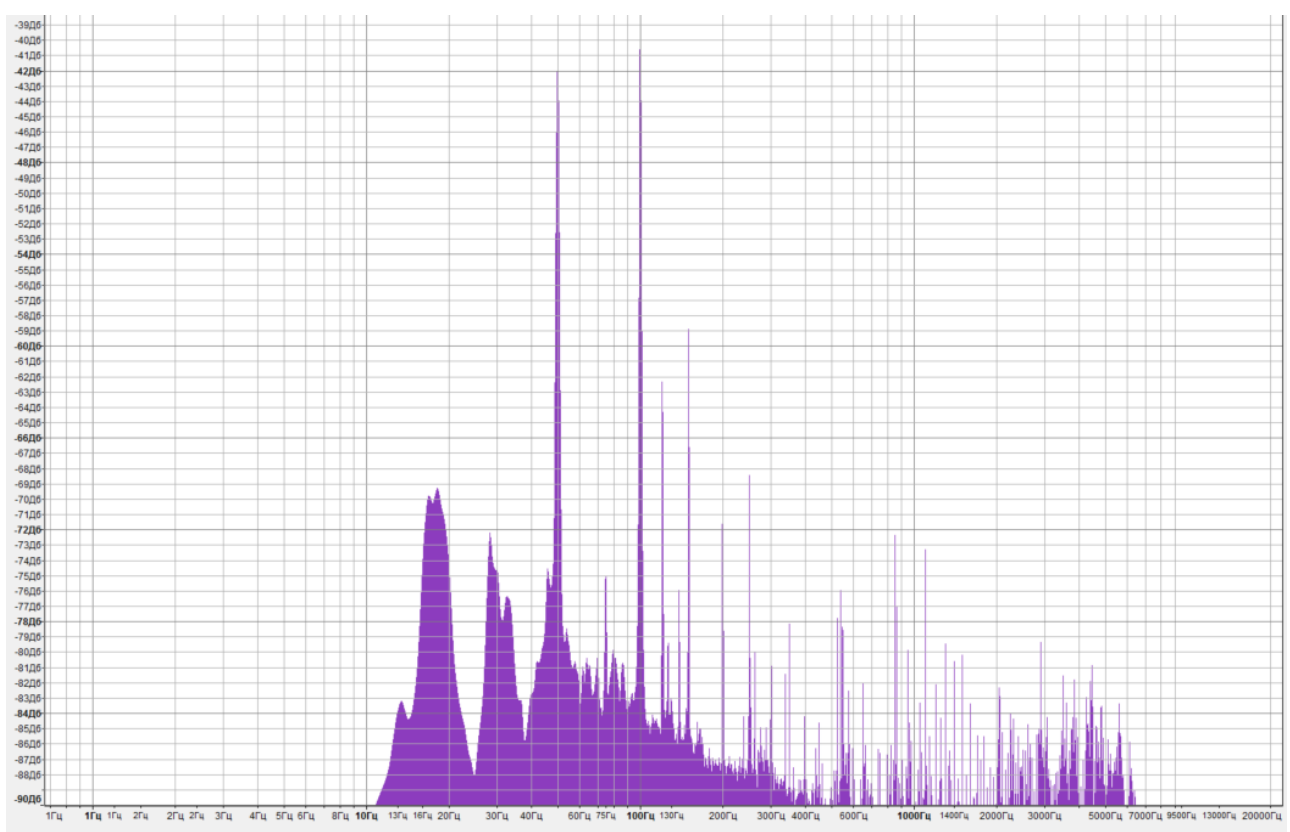

c

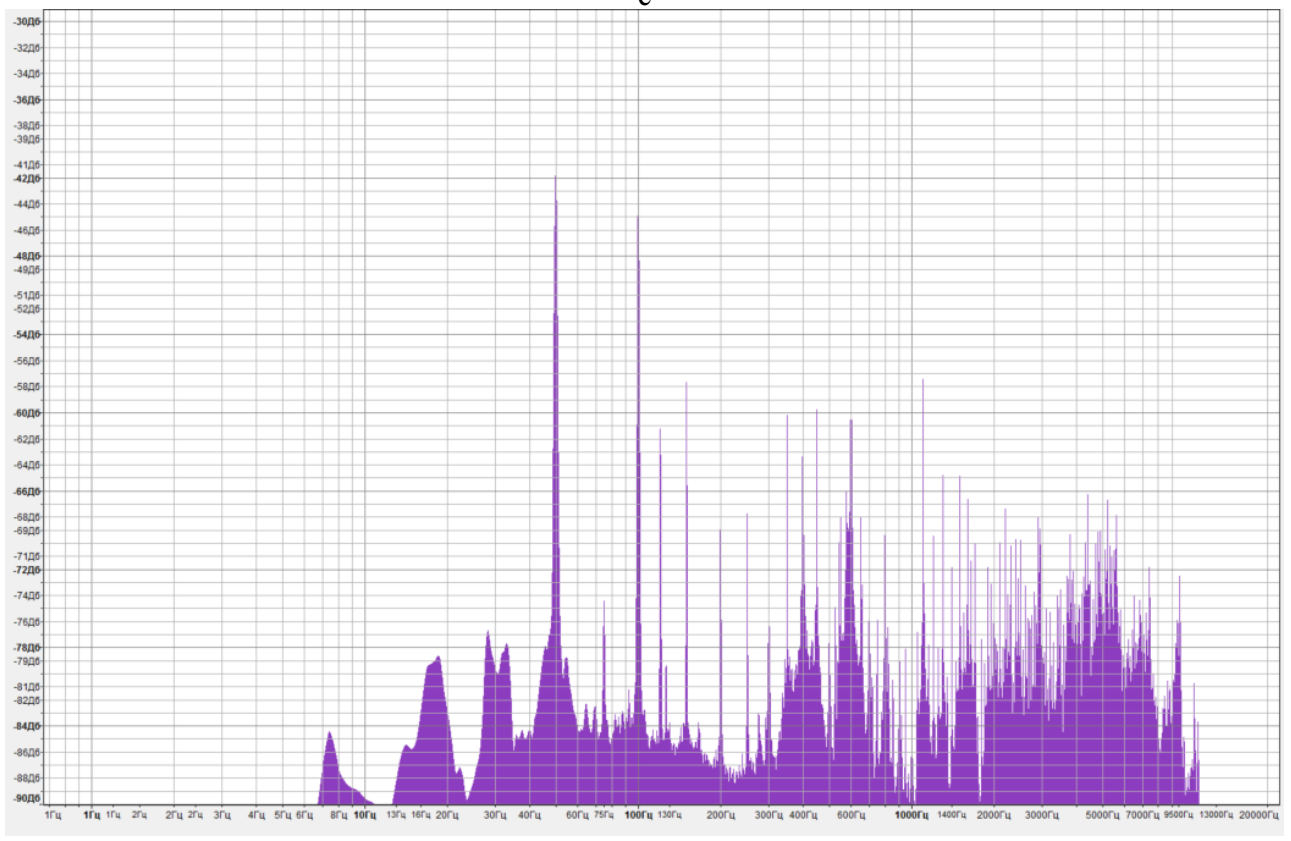

d

Fig. 5. Fourier spectra of acoustic emission signals recorded on the 1st (a), 15 (b), 55 (c) and 88 minutes (d) minutes of testing.

In the run mode of the connection (Fig. 5 b, c) is characterized by a substantial weakening of the high frequency and midrange components of the acoustic spectrum, show a decrease in the initial rate of microplastic deformation. General view of the spectra in this case is close to the mean spectra of the idle mode of operation.

A qualitative change in the spectral composition of the acoustic emission of friction associated with the violation of the integrity of the connection is clearly seen in the 
spectrum of Fig. 5 d. Here again amplified high frequency components, and in addition to this there are two strong mid-frequency components of the $\approx 400 \mathrm{~Hz}$ and about $600 \mathrm{~Hz}$. The latter is necessary to characterize the modes of the contact gap, because their appearance is directly related to the wear of units, with a corresponding decrease in the contact stiffness and slippage in the contact.

Thus, use a public domain sound recording equipment and standard computer software allows, at least with respect to low-noise experimental installations, to carry out contactless acoustic control of the integrity of nominally stationary friction joints under conditions of fretting. Violation of the integrity of such joints is recorded as a change in the overall shape of ecostorm operating noise, and (more reliably) - characteristic changes in the spectrum of sound with a strong mid-frequency components of the spectrum arising from the contact gaps.

Low frequency self-modulation of the friction process, which is manifested in the spectrum of the acoustic emission is a consequence of the dynamic targeting slow angular motion in the system friction. A-modulation is a mistake to ascribe directly of cyclical damage accumulation and separation of wear particles.

\section{Conclusions}

The use of triboacoustic monitoring the fretting is practical for the real-time detection of a disturbance in the integrity of nominally motionless friction joints. This disturbance was determined by the change in the spectrum of the acoustograms of working noise, as well as by the characteristic changes in the sound spectrum due to the appearance of strong midfrequency components caused by the occurrence of contact gaps. Low-frequency friction self-modulation results from the dynamic generation of slow angular motions in the friction system during the reverse energy cascade and is directed from the pumping modes toward low frequencies, which manifests in acoustograms and the spectrum of acoustic emission. In parallel with this, the direct energy cascade (toward high frequencies) is due to plastic deformations in the zones of the actual joint contact.

\section{References}

1. A. Sviridenok, N. Myshkin, T. Kalmykova and O. Kholodilov. Acoustic and Electrical Methods in Tribotechnics, Minsk, Nauka i Tekhnika (1987)

2. A. Akay, J. Acoust. Soc. Am., V. 111, 4 (2002)

3. V. Vlasov, N. Mel'nichenko and E. Reizer, Trenie Iznos, 1989, V. 10, 2 (1989)

4. Yu. Fadin, A. Leksovskii, B. Ginzburg and V. Bulatov, Pis'ma Zh. Tekh. Fiz., V. 19, 5 (1993)

5. B. Gritsenko, Trenie Iznos, V. 26, no. 5, (2005)

6. A. Kolubaev, E. Kolubaev, I. Vagin and O. Sizova, Tech. Phys. Lett., V. 31, 10, (2005)

7. V. Rubtsov, E. Kolubaev, A. Kolubaev and V. Popov, Tech. Phys. Lett., V. 39, 2, (2013)

8. V. Zaporozhets and V. Stadnichenko, J. Frict. Wear, V. 36, 3, (2015)

9. A. Dykha, and A. Kuz'menko, J. Frict. Wear, V. 37, 4, (2016)

10. V. Kurskoi, V. Slashchuk and A. Slashchuk, UA Patent 94006, Byull. Izobret. 20, (2014) 